\title{
Exercícios físicos de alto impacto e incontinência urinária em mulheres jovens: revisão integrativa da literatura
}

\author{
High-impact physical exercise and urinary incontinence in young women: an integrative literature \\ review
}

\author{
Luciana Gomes de Souza Camelo ${ }^{1}$, Raphael Fernando Soares Gonçalves ${ }^{2} \bullet$, Tânia Aparecida Barbosa \\ Rzniski $^{2}$
}

\begin{abstract}
${ }^{1}$ Fisioterapeuta, Faculdade Paranaense, Curitiba, Paraná, Brasil. ${ }^{2}$ Docente do Curso de Graduação em Fisioterapia, Faculdade Paranaense, Curitiba, Paraná, Brasil. *Autor para correspondência. E-mail: luciana.vandinha@hotmail.com
\end{abstract}

\begin{abstract}
Resumo: Introdução: A Incontinência urinária de esforço (IUE) é definida como a queixa de perda involuntária de urina por meio de esforço físico, espirro ou tosse. O estudo teve por objetivo compreender a incidência de incontinência urinária relacionada a prática de exercícios de alto impacto em mulheres jovens. Método: Foi realizado um estudo exploratório descritivo, desenvolvido a partir do método de revisão integrativa. Foram selecionados artigos científicos publicados entre o período de 2020 e 2021. Dos 88 estudos avaliados, 10 atenderam aos critérios de inclusão e exclusão. Identificou-se que as idades das participantes variaram consideravelmente, de forma que a média de idade das participantes variou de 18 anos até 43 anos de idade. A média de população por estudo ficou estimada em 119,9 mulheres por estudo analisado. Revisão: Os resultados coletados foram relacionados a mulheres que praticavam atividades físicas, tais como ginástica rítmica, CrossFit, BodyPump, incluindo na amostra mulheres que estavam em processo de retomada de atividades físicas pós-parto. Não foi identificado que atividades físicas de alto impacto são a causa direta de IUE. Verificou-se correlação entre mulheres praticantes de atividades físicas com impacto e a incidência deste tipo de incontinência. Não existem tratamentos convencionados e totalmente indicados para essa patologia em mulheres do sexo feminino que pratiquem esportes. Considerações finais: $\mathrm{O}$ treinamento de fortalecimento de músculos do assoalho pélvico foi relatado nos estudos com taxas promissoras para o controle de IUE, não tendo efeitos adversos conhecidos, podendo desta forma ser oferecido às mulheres praticantes destas atividades.
\end{abstract}

Palavras-chaves: incontinência urinária, incontinência urinária por estresse, exercício físico e fisioterapia.

\begin{abstract}
Introduction: Stress urinary incontinence (SUI) is defined as the complaint of involuntary loss of urine through physical exertion, sneezing or coughing. The study aimed to understand a treatment for urinary incontinence related to high-impact exercise practice in young women. Method: A descriptive exploratory study was carried out, developed from the integrative review method. Scientific articles published between the period 2020 and 2021 were selected. Of the 88 approved studies, 10 met the inclusion and exclusion criteria. It was identified that the ages of the participants varied considerably, so that the average age of the participants ranged from 18 years old to 43 years old. The mean of the population per study was evaluated at 119.9 women per study analyzed. Review: The results collected were related to women who practiced physical activities, such as rhythmic gymnastics, CrossFit, BodyPump, including in the sample women who were in the process of resuming physical activities after delivery. It was not identified that high-impact physical activities are the direct cause of SUI. There was a correlation between women who practice physical activities with impact and the incidence of this type of incontinence. There are no conventional and fully indicated treatments for this pathology in female women who practice sports. Final considerations: The training of pelvic tissue muscle strengthening was reported in studies with promising rates for the control of SUI, with no known adverse effects, and may thus be for women who practice these activities.
\end{abstract}

Keywords: urinary incontinence, effort urinary incontinence, exercise, physical therapy specialty.

\section{Introdução}

A Sociedade Internacional de Continência (ICS), define a incontinência urinária como sendo, “a queixa de qualquer perda involuntária de urina”, estando entre as grandes síndromes geriátricas (Rådmark et al., 2015). 
Segundo o estudo de Kargar et al. (2014), sobre a micção excessiva ou frequente, constatou-se como sendo desconfortável em relação aos aspectos sociais e higiênicos.

No que tange a IU e sua prevalência na população, identificou-se que entre $8 \%$ a $34 \%$ das pessoas com mais de 65 anos apresentem algum grau de incontinência urinária, sendo o maior percentual encontrado no sexo feminino. O estudo de Henkes et al. (2015) aponta que 10,7\% das mulheres no Brasil procuraram tratamento ginecológico em decorrência da perda urinária.

Com relação a sua classificação, existem três tipos de incontinência urinária (IU): incontinência urinária de esforço (IUE), incontinência urinária de urgência (IUU) e incontinência urinária mista (IUM). A IU em mulheres, está relacionada a diversos fatores de risco, como idade, hereditariedade, obesidade, raça, menopausa, consumo excessivo de cafeína, tabagismo, dentre outros (Higar, Lopes, \& Reis, 2008).

Já nos aspectos que envolvem a IU e sua prevalência na população, identificou-se que entre $8 \%$ a $34 \%$ das pessoas com mais de 65 anos apresentem algum grau de incontinência urinária, sendo o maior percentual encontrado no sexo feminino. O estudo de Henkes et al. (2015) aponta que 10,7\% das mulheres no Brasil procuraram tratamento ginecológico em decorrência da perda urinária.

No entanto, estudos revelaram que a experiência com episódios de perda urinária já não é mais um problema exclusivo do envelhecimento. Com isso, a prática de exercícios físicos de alto impacto tem a capacidade de influenciar na saúde de mulheres jovens, contribuindo com o desenvolvimento de incontinência urinária (Santos et al., 2020).

A IUE é definida como a queixa de perda involuntária de urina por meio de esforço físico, espirro ou tosse (Abrams et al., 2003), apresentando maior prevalência dentre os tipos de IU, ocorrendo de $12 \%$ a $56 \%$ dependendo do grupo estudado e de critérios utilizados para o diagnóstico (Hannestad et al., 2000). Por sua vez, a IUU é considerada como a perda involuntária de urina que se associa ao desejo súbito de urinar, podendo acontecer de forma espontânea ou desencadeada por eventos como mudança súbita de temperatura, estresse, emoções ou situações de medo ou perigo (Haylen et al., 2010). Já a IUM é compreendida como a queixa de perda involuntária de urina motivada pelo esforço físico, assim como posteriormente a uma situação de urgência (Borba et al., 2008).

De acordo com Patrizzi et al. (2014), a IUE é a de maior prevalência no sexo feminino, oscilando entre $12,6 \%$ a $48,0 \%$ e a prevalência geral de IU em mulheres é entre 8,5 a $55 \%$. De acordo com o estudo, existe uma prevalência de IUE de $26,5 \%$ em mulheres jovens sendo que esses números aumentam, passando para $41 \%$ conforme o avanço da idade.

Exercícios de alto impacto são definidos como um conjunto de exercícios aeróbios e anaeróbicos, com a finalidade de melhorar o condicionamento físico do atleta (Nygaard et al., 2005). Essa abordagem tem o objetivo de levar o atleta ao seu limite a partir de exercícios como agachamentos, arremessos e desenvolvimentos, exercícios aeróbicos, corrida, bicicleta bem como movimentos ginásticos como paradas de mão, paralelas, argolas e barras (Caetano et al., 2007).

Segundo Khaida (2018) a execução de exercícios de alto impacto decorre na contenção da musculatura do assoalho pélvico em função dos aumentos súbitos da pressão intra-abdominal. Os exercícios de alto impacto são normalmente realizados em três etapas, o aquecimento, o desenvolvimento de técnica e o "treino do dia", expressão do inglês "workout of the day" (Tibana, Almeida, \& Prestes, 2015). Exercícios de alto impacto exigem maior recrutamento das funções de suporte e contenção da musculatura do assoalho pélvico e, uma vez que exista a ausência ou déficit dessas funções podem ocorrer episódios de perda urinária, configurando a IUE, uma vez que estes perfis de modalidade de atividade física normalmente possuem movimentos e exercícios que podem apresentar riscos à saúde da mulher, relacionado a saltos, arranques e movimentos rápidos (Khaida, 2018).

Para Araújo et al., (2015), a maior parte dos exercícios nos quais existe o aumento da pressão intraabdominal não ocorre contração voluntária dos músculos do assoalho pélvico, o qual é compreendido como um conjunto de músculos e ligamentos responsável pela sustentação de órgãos pélvicos como bexiga, útero, reto e intestino, o que pode justificar a perda involuntária de urina durante a prática de alguns esportes. De acordo com Casey e Temme (2017), o assoalho pélvico fornece suporte adequado para os órgãos pélvicos, de modo que a contração e o relaxamento dessa região devem ocorrer de maneira coordenada, consciente ou inconsciente, assegurando resultados desejados de continência ou vazamento.

Conforme relatado por Almeida e Machado (2012) e Caetano et al. (2007), mulheres jovens fisicamente ativas possuem maior incidência de IU durante a prática de exercícios que exigem maior esforço físico, principalmente aqueles que caracteristicamente possuem alto impacto. Sendo assim, embora ainda não exista consenso na literatura sobre qual a taxa de prevalência de IUE em mulheres com relação a faixa etária, alguns estudos, como o de Azevedo (2013), indicam que existe prevalência de IUE em mulheres jovens, 
especificamente aquelas que praticam esportes de alto impacto nos quais existem movimentos envolvendo saltos.

A prática de exercícios físicos de alto impacto e o risco de desenvolvimento de IU em mulheres jovens vem sendo nos últimos anos investigadas (Zago; Silva; Lopes, 2018), buscando avaliar a incidência em mulheres que praticam atividades físicas como o Jump, Basquetebol, Voleibol, Handebol, CrossFit e atletismo (Nygaard \& Shaw, 2017).

Uma vez que esta temática ainda é explorada pela literatura de forma incipiente, se fazendo pertinente o desenvolvimento de novas pesquisas que tenham a finalidade de compreender a relação entre a IU e a prática esportiva de mulheres jovens. Diante disso, o objetivo do presente estudo consiste em compreender a incidência de incontinência urinária relacionada a prática de exercícios de alto impacto em mulheres jovens.

\section{Revisão}

\section{Métodos}

O presente artigo científico compreende um estudo exploratório descritivo, o qual foi desenvolvido a partir do método de revisão integrativa. A partir disso, a questão norteadora deste estudo foi: "A prática de exercícios físicos de alto impacto pode favorecer a incontinência urinária em mulheres jovens?"

Para a construção da pergunta de pesquisa fora utilizado o acrônimo PICO (P - População; I - Intervenção; C - Comparação; O - Resultados, no qual: P - Mulheres Jovens; I - Exercícios de alto impacto ; C - Exercícios físicos de alto impacto $\mathrm{x}$ exercícios físicos sem impacto; $\mathrm{O}$ - Melhores práticas e condutas no atendimento dos fisioterapeutas.

A coleta de dados foi realizada a partir de artigos científicos publicados nos idiomas português e inglês a partir das bases de dados Scientific Eletroctronic Library Online - SCIELO, MEDLINE/PubMed, PEDro, Periódicos CAPES/MEC e Cochrane Library entre abril e maio de 2021, com critério temporal para ano de publicação entre os anos de 2016 a 2021. Definiu-se os descritores definidos de acordo com o Descritores em Ciências da Saúde - DeCs: Em português - Incontinência Urinária, Incontinência Urinária por Estresse, Exercício Físico e Fisioterapia. Em inglês - Urinary Incontinence, Effort Urinary Incontinence, Exercise, Physical Therapy Specialty. O operador booleano utilizado foi AND.

Baseado nos critérios de inclusão do estudo, optou-se por estudos primários, os quais são correspondentes às pesquisas originais (Polit \& Beck, 2011), delimitando-se para relatos de casos, estudos de caso e de controle. A coleta desse material ocorreu eletronicamente, de forma correspondente à questão norteadora do estudo. Como critérios de exclusão de estudo foi estabelecido que estudos secundários, sendo esses estudos pesquisas de revisões sistemáticas de conteúdo, revisões não sistemáticas, metanálises, dissertações, teses e demais estudos que se distinguiam da proposta desta investigação.

\section{Resultados}

A seguir são apresentados os resultados do estudo desenvolvido. Inicialmente, a figura 1 apresenta o fluxograma de seleção de estudos do PRISMA correspondente as etapas de revisão da literatura, descrevendo todos os portais de consulta de artigos científicos utilizados no estudo e discriminando o processo de seleção do material analisado.

Dos 88 artigos encontrados incluídos pelos critérios de palavras-chaves, 10 atenderam aos critérios de inclusão e exclusão. Abaixo, na tabela 2, são apresentadas as principais informações coletadas que identificam os artigos analisados a partir do ano de publicação, autores, título da pesquisa, principais objetivos e desfechos do estudo.

Na tabela 2 apresenta-se a caracterização dos artigos científicos selecionados para compor a revisão integrativa da literatura. Nessa tabela estão discriminados os autores do estudo, ano de publicação, idioma de publicação do estudo, método utilizado, número da amostra e o público-alvo do estudo desenvolvido.

Foram analisados dez artigos científicos com período de publicação variando entre os anos de 2020 e 2021 . Do total de dez artigos selecionados, predominaram para a amostra final artigos redigidos originalmente, publicado em inglês $(n=9)$ e português $(n=1)$. O local de publicação dos artigos científicos foi, respectivamente, nos seguintes países Estados Unidos da América $(n=1)$, Europa $(n=7)$, Austrália $(n=1)$ e Brasil $(n=1)$. A natureza dos artigos científicos analisados foi identificada como longitudinal $(n=3)$, transversal $(n=4)$, retrospectiva $(n=2)$, controlada $(n=1)$ e semiqualitativa $(n=1)$, onde $" n="$ corresponde ao número de artigos verificados que correspondem à natureza de pesquisa mencionada.

Nos estudos analisados, as idades das participantes variaram consideravelmente, de forma que a média de idade das participantes variou de 18 anos até 43 anos de idade. A média de população por estudo ficou 
estimada em 119,9 mulheres por estudo analisado. No que tange as atividades físicas praticadas no estudo, verificou-se que os estudos tinham como amostra mulheres ginastas rítmicas competidoras de alto nível, praticantes de CrossFit, atletas de CrossFit, mulheres atletas de alto nível, mulheres retomando as atividades físicas pós-parto, mulheres jovens e mulheres com sobrepeso que praticavam BodyPump.

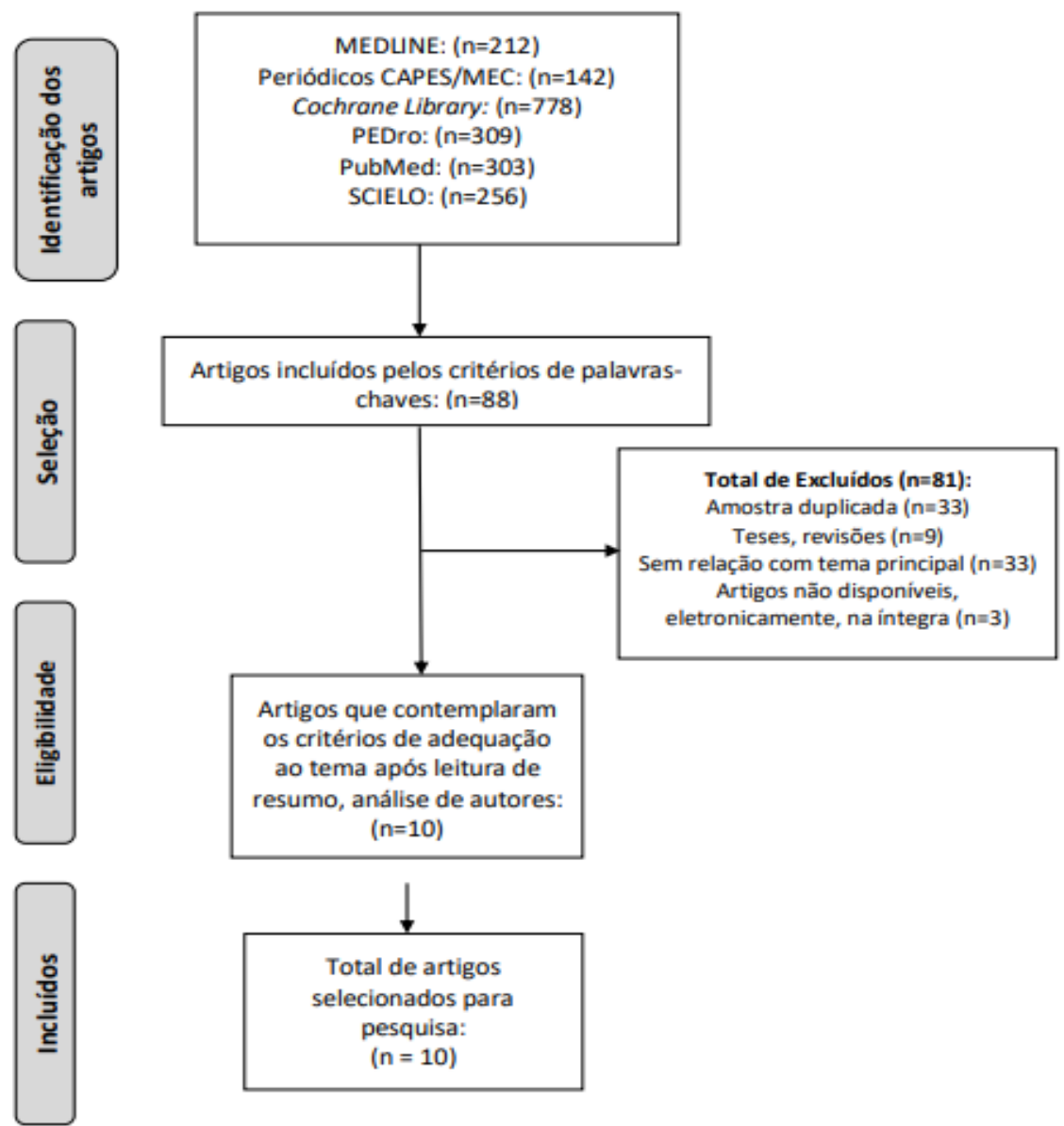

Figura 1. Fluxograma de coleta de dados e seleção de estudos que compõe a amostra

Tabela 1. Apresentação das principais informações analisadas nos artigos selecionados.

\begin{tabular}{|c|c|c|c|c|}
\hline Ano & Autores & Título & Objetivo & Desfecho \\
\hline 2020 & $\begin{array}{l}\text { Khowailed } \\
\text { et al. }\end{array}$ & $\begin{array}{l}\text { Stress Incontinence during } \\
\text { Diferent High-Impact } \\
\text { Exercises in Women: A Pilot } \\
\text { Survey }\end{array}$ & $\begin{array}{l}\text { Avaliar a incidência de IUE } \\
\text { em mulheres fisicamente } \\
\text { ativas e examinar exercícios } \\
\text { específicos de alto impacto. }\end{array}$ & $\begin{array}{l}\text { Identificados sintomas de IU em mulheres primigestas. } \\
\text { Mulheres saudáveis fisicamente ativas que praticam } \\
\text { exercícios de alto impacto devem estar cientes de que } \\
\text { pode existir um risco maior de IUE. }\end{array}$ \\
\hline 2020 & Kari e Gram. & $\begin{array}{l}\text { High level rhythmic gymnasts } \\
\text { and urinary incontinence: } \\
\text { Prevalence, risk factors, and } \\
\text { influence on performance }\end{array}$ & $\begin{array}{l}\text { Investigar a prevalência e os } \\
\text { fatores de risco para IU em } \\
\text { ginastas rítmicas e o impacto } \\
\text { da IU no desempenho de } \\
\text { atletas. }\end{array}$ & $\begin{array}{l}\text { A prevalência de IU em ginastas jovens nulíparas } \\
\text { rítmicas está acima de } 30 \% \text { e } 70 \% \text {. As condições que } \\
\text { estão associadas a ocorrência de problemas e a IU são } \\
\text { estresse, urgência, incontinência urinária mista e } \\
\text { vazamento sem motivo óbvio. }\end{array}$ \\
\hline 2020 & $\begin{array}{l}\text { Wikander et } \\
\text { al. }\end{array}$ & $\begin{array}{c}\text { Urinary Incontinence and } \\
\text { Women CrossFit Competitors }\end{array}$ & $\begin{array}{l}\text { Determinar a prevalência de } \\
\text { incontinência urinária e } \\
\text { atlética e estabelecer quais } \\
\text { atividades e contextos eram } \\
\text { mais prováveis de provocar } \\
\text { perda de urina em mulheres } \\
\text { competidoras de CrossFit. }\end{array}$ & $\begin{array}{l}\text { A incontinência urinária foi experimentada por } 41,8 \% \\
\text { dos participantes nos três meses anteriores ao estudo e } \\
\text { 17,7\% experimentaram incontinência atlética. } 8 \% \text { das } \\
\text { mulheres eram incontinentes antes de iniciar o CrossFit, } \\
\text { agora são continentes durante as atividades diárias, mas } \\
\text { permanecem incontinentes durante os exercícios. }\end{array}$ \\
\hline 2021 & Kari et al. & $\begin{array}{l}\text { Does regular strength training } \\
\text { cause urinary incontinence in } \\
\text { overweight inactive women? A } \\
\text { randomized controlled trial }\end{array}$ & $\begin{array}{l}\text { Investigar o novo início de } \\
\text { IU em mulheres } \\
\text { anteriormente inativas, com } \\
\text { sobrepeso ou obesas (IMC> } \\
25 \text { ). }\end{array}$ & $\begin{array}{l}\text { Cerca de } 31,2 \% \text { participantes relataram IU. Não houve } \\
\text { diferenças estatisticamente significativas na nova IU de } \\
\text { início entre os grupos ou quando o colapso da nova IU } \\
\text { de início nos grupos de intervenção em comparação com } \\
\text { os controles. }\end{array}$ \\
\hline
\end{tabular}




\begin{tabular}{|c|c|c|c|c|}
\hline 2020 & $\begin{array}{l}\text { Campbell, } \\
\text { Batt, \& } \\
\text { Drummond }\end{array}$ & $\begin{array}{l}\text { A feasibility study of the } \\
\text { physiotherapy management of } \\
\text { urinary incontinence in } \\
\text { athletic women: trial protocol } \\
\text { for the POsITIve study }\end{array}$ & $\begin{array}{l}\text { Investigar a viabilidade de } \\
\text { conduzir um o manejo } \\
\text { fisioterapêutico da IU em } \\
\text { mulheres atletas. }\end{array}$ & $\begin{array}{c}\text { As mulheres atléticas e que praticam exercícios } \\
\text { regularmente, recrutadas diretamente de academias e } \\
\text { clubes esportivos, devem realizar sessões de fisioterapia } \\
\text { para lidar com a IU. }\end{array}$ \\
\hline 2021 & $\begin{array}{c}\text { Rzymski et } \\
\text { al. }\end{array}$ & $\begin{array}{l}\text { How to balance the treatment } \\
\text { of stress urinary incontinence } \\
\text { among female athletes? }\end{array}$ & $\begin{array}{l}\text { Avaliar os tratamentos } \\
\text { existentes para a } \\
\text { incontinência urinária de } \\
\text { esforço entre atletas do sexo } \\
\text { feminino. }\end{array}$ & $\begin{array}{l}\text { Relata que a ultrassonografia do assoalho pélvico é o } \\
\text { único método objetivo para o exame do assoalho pélvico } \\
\text { entre esportistas e uma ferramenta que deve ser usada } \\
\text { rotineiramente por urofisioterapeutas e } \\
\text { uroginecologistas. }\end{array}$ \\
\hline 2020 & $\begin{array}{l}\text { Mazur-Bialy } \\
\text { et al. }\end{array}$ & $\begin{array}{l}\text { Urinary Incontinence in } \\
\text { Women: Modern Methods of } \\
\text { Physiotherapy as a Support } \\
\text { for Surgical Treatment } \\
\text { orIndependent Therapy }\end{array}$ & $\begin{array}{c}\text { Realizar uma revisão sobre } \\
\text { as possibilidades de } \\
\text { utilização de técnicas } \\
\text { fisioterapêuticas no } \\
\text { tratamento da IU em } \\
\text { mulheres com atenção às } \\
\text { técnicas de ativação dos } \\
\text { MAP. }\end{array}$ & $\begin{array}{l}\text { A fisioterapia é um elemento importante no preparo do } \\
\text { paciente para o tratamento cirúrgico, aumentando } \\
\text { significativamente o sucesso da cirurgia. }\end{array}$ \\
\hline 2020 & Hagen et al. & $\begin{array}{l}\text { Basic versus biofeedback- } \\
\text { mediated intensive pelfic floor } \\
\text { muscle training: for women } \\
\text { with urinary incontinence: the } \\
\text { OPAL RCT }\end{array}$ & $\begin{array}{l}\text { Determinar a eficácia clínica } \\
\text { e custo-benefício de } \\
\text { diferentes métodos para o } \\
\text { tratamento de IUE. }\end{array}$ & $\begin{array}{l}\text { O treinamento dos músculos do assoalho pélvico com } \\
\text { biofeedback foi não significativamente mais caro do que } \\
\text { o treinamento muscular básico do assoalho pélvico, mas } \\
\text { também não estava associado a significativamente mais } \\
\text { anos de vida ajustados pela qualidade. }\end{array}$ \\
\hline 2020 & Bógea et al. & $\begin{array}{l}\text { Incontinência Urinária de } \\
\text { Esforço em Mulheres } \\
\text { Praticantes de CrossFit: Um } \\
\text { estudo transversal de } \\
\text { prevalência }\end{array}$ & $\begin{array}{l}\text { Verificar a prevalência de } \\
\text { incontinência urinária de } \\
\text { esforço em mulheres } \\
\text { praticantes de CrossFit. }\end{array}$ & $\begin{array}{l}\text { A intervenção intensificada em ambos os grupos } \\
\text { recebeu componentes básicos do núcleo de treinamento } \\
\text { dos músculos do assoalho pélvico. As mulheres foram } \\
\text { positivas sobre ambas as intervenções. }\end{array}$ \\
\hline 2020 & $\begin{array}{l}\text { Sorrigueta- } \\
\text { Hernández } \\
\quad \text { et al. }\end{array}$ & $\begin{array}{l}\text { Benefits of Physiotherapy on } \\
\text { Urinary Incontinence in High- } \\
\text { Performance Female Athletes. } \\
\text { Meta-Analysis }\end{array}$ & $\begin{array}{l}\text { Identificar as evidências } \\
\text { científicas para disfunções } \\
\text { do assoalho pélvico (FP) } \\
\text { associadas à incontinência } \\
\text { urinária (IU) em esportistas } \\
\text { de alto rendimento e } \\
\text { determinar se a fisioterapia } \\
\text { do assoalho pélvico (TP) } \\
\text { corrige a IU em atletas de } \\
\text { elite do sexo feminino. }\end{array}$ & $\begin{array}{l}\text { Em atletas femininas de elite a fisioterapia contribuiu } \\
\text { para o ganho de continência urinária mais do que em } \\
\text { mulheres controle. O ganho de IU em atletas de elite do } \\
\text { sexo feminino, ex-atletas de elite do sexo feminino e em } \\
\text { mulheres grávidas que se envolvem regularmente em } \\
\text { atividades aeróbicas prevaleceu do que se em relação ao } \\
\text { grupo controle. }\end{array}$ \\
\hline
\end{tabular}

Tabela 2. Apresentação das principais informações dos artigos selecionados.

\begin{tabular}{|c|c|c|c|c|c|c|}
\hline Autores & Ano & Local & Idioma & Método & $\mathbf{n}$ & Público-alvo \\
\hline Khowailed et al. & 2020 & $\begin{array}{l}\text { Califórnia, } \\
\text { EUA }\end{array}$ & Inglês & $\begin{array}{l}\text { Ensaio clínico } \\
\text { Controlado } \\
\text { randomizado ou } \\
\text { aleatório }\end{array}$ & 17 & $\begin{array}{c}\text { Mulheres que passaram primigesta, } \\
\text { multigravida e nuligesta }\end{array}$ \\
\hline Kari e Gram. & 2020 & Oslo, NO & Inglês & $\begin{array}{l}\text { Estudo transversal } \\
\text { prospectivo }\end{array}$ & 133 & $\begin{array}{l}\text { Mulheres ginastas rítmicas } \\
\text { competidoras de alto nível } \\
\text { nacional e internacional }\end{array}$ \\
\hline Wikander et al. & 2020 & $\begin{array}{c}\text { Northern } \\
\text { Territory, } \\
\text { AU }\end{array}$ & Inglês & $\begin{array}{l}\text { Estudo } \\
\text { transversal e } \\
\text { bibliográfico }\end{array}$ & 452 & Mulheres atletas de CrossFit \\
\hline Kari et al. & 2021 & Oslo, NO & Inglês & $\begin{array}{l}\text { Estudo de análise } \\
\text { secundária }\end{array}$ & 1 & Mulheres atletas ginastas \\
\hline $\begin{array}{l}\text { Campbell, Batt, \& } \\
\text { Drummond }\end{array}$ & 2020 & Nottingham, UK & Inglês & $\begin{array}{l}\text { Estudo com métodos } \\
\text { mistos: entrevistas } \\
\text { semi-estruturadas e } \\
\text { análise clínica }\end{array}$ & 8 & $\begin{array}{l}\text { Mulheres atletas que praticam } \\
\text { exercícios regularmente }\end{array}$ \\
\hline Rzymski et al. & 2021 & Katowice, PO & Inglês & $\begin{array}{l}\text { Estudo qualitativo } \\
\text { multidisciplinar } \\
\text { individualizado }\end{array}$ & 143 & $\begin{array}{c}\text { Mulheres com sobrepeso, inativas, } \\
\text { que iniciaram a prática de } \\
\text { BodyPump }\end{array}$ \\
\hline
\end{tabular}




\begin{tabular}{lcccccc}
\hline Mazur-Bialy et al. & 2020 & Cracóvia, PO & Inglês & Estudo transversal & 32 & Mulheres atletas \\
\hline Hagen et al. & 2021 & Perth, SLD & Inglês & $\begin{array}{c}\text { Estudo controlado } \\
300\end{array}$ & $\begin{array}{c}\text { Mulheres com mais de 18 anos de } \\
\text { idade }\end{array}$ \\
\hline $\begin{array}{l}\text { Bógea et al. } \\
\text { Sorrigueta- } \\
\text { Hernández et al. }\end{array}$ & 2020 & $\begin{array}{c}\text { Rio de Janeiro, } \\
\text { BR }\end{array}$ & Português & Estudo transversal & 91 & Mulheres praticantes de CrossFit \\
\hline
\end{tabular}

\section{Discussão}

A IUE é considerada pelos artigos científicos estudados como o tipo mais comum de IU, sendo caracterizada por sintomas como a perda de urina ao tossir e/ou espirrar e em conformidade com a frequência de impacto sobre o períneo, sendo esse fato comum durante a realização de atividades físicas de alto impacto que implicam no esforço físico de mulheres (Wikander et al., 2020; Khowailed et al., 2020; Campbell, Batte, \& Drummond, 2020; Sorrigueta-Hernandez et al., 2020).

Para Araújo et al. (2015) e Frigo et al. (2015) a IUE compromete diversas esferas da vida de uma mulher, impactando em suas atividades diárias, interações sociais e também no como como essas mulheres percebem sua saúde, o qual é afetado de forma significativa, comprometendo negativamente a qualidade de vida de mulheres. A IUE pode gerar danos psicológicos, físicos, sexuais, profissionais e sexuais à vida da mulher. Para Padilha et al. (2018) a IU é uma das doenças crônicas de alta prevalência no público feminino. Nessa perspectiva, é possível perceber que a IU é um fenômeno que acomete as mulheres com alta incidência, comprometendo a sua qualidade de vida e, não necessariamente, está relacionada a hábitos alimentares, horários de treinamento, mobilidade e IMC.

Para Wikander et al. (2020) a incontinência urinária é uma condição comum que potencialmente desestimula as mulheres de participarem de atividades físicas. Conforme o estudo, as atividades CrossFit de alto impacto foram mais propensas a provocar incontinência urinária. Khowailed et al. (2020) mulheres praticantes da modalidade CrossFit foram significativamente mais propensas a relatar vazamento urinário do que atletas de esportes como kickboxing e bootcamp combinados. Nessa perspectiva, é possível apontar que as mulheres que fazem exercícios de alto impacto são suscetíveis à IUE e que o CrossFit representa um risco maior de IUE em termos de mais saltos, resultando em aumento da pressão intra-abdominal e forças de reação do solo em comparação para outros.

O estudo realizado por Kari e Gram (2020) sinaliza que não foi identificada relação entre a IUE e fatores como o Índice de Massa Corporal - IMC, hipermobilidade, alimentação desordenada e horas de treinamento, de forma que esses aspectos não foram considerados fatores de risco para incontinência urinária de esforço. Conforme o estudo, existe prevalência de IU em ginastas jovens nulíparas rítmicas, de modo que esta condição teve alguma influência no desempenho esportivo.

Para Silva et al. (2020) a atividade física é um fator de risco modificável, o qual está associado a prevalência de IUE. Todavia, os autores destacam que não foram identificadas relações entre as atividades físicas e ocorrência de IUE. Nessa perspectiva, é importante se atentar que diferentes populações podem apresentar índices de prevalência de IU e IUE diferenciadas. Conforme verificado no estudo, a IUE tende a prevalecer mais sobre populações que praticam modalidades onde prevalecem movimentos de saltos de alto impacto, exercícios que poderiam agravar quadros de IU.

Por outro lado, de acordo com Martins et al. (2017), no treinamento de força, exercícios que trabalhem o fortalecimento de músculos do assoalho pélvico são, em muitos casos, deixados de lado em algumas atividades físicas. Se tratando de esportes tais como os de ginástica, esse fato não é coerente com o próprio esporte, uma vez que são os músculos do assoalho pélvico que fornecem a capacidade de mulheres desenvolverem com êxito suas atividades funcionais.

A pesquisa de Leal et al. (2020) também identificou baixa prevalência de IUE em atletas praticantes de CrossFit. Por sua vez, os autores apontam que informações sociodemográficas, antecedentes obstétricos e nível de performance das mulheres analisadas podem interferir diretamente nos resultados obtidos pelo estudo. Porém, nas mulheres que positivaram a incidência de IU, foi relatado que a IU pode comprometer atividades cotidianas, ritmo de treino e interação social.

De acordo com o estudo de Silva et al. (2020) a IU pode prejudicar seriamente a qualidade de vida e saúde das mulheres. Seu tratamento deve ser realizado por meio de identificação precoce, verificando-se sua 
classificação e gravidade. A IU deve ser tratada por médicos generalistas, enfermeiros e fisioterapeutas. Conforme o estudo, o fortalecimento do assoalho pélvico é uma forma de prevenir a IU.

Nesse sentido, para Campbell, Batt e Drummond (2020) mulheres atléticas e que praticam exercícios regularmente, recrutadas diretamente de academias e clubes esportivos, devem ter acesso a fisioterapia para lidar com a IU. Saboia et al. (2017) concorda com Campebell, Batte e Drummond (2020), apontando que se faz necessária a intervenção por meio de fortalecimento do assoalho pélvico em pacientes com IUE, e que todos os tipos de incontinência urinária podem afetar diretamente e de forma negativa a qualidade de vida das mulheres. Como principais problemas apresentados no estudo, cita-se as limitações na vida diária das pacientes.

Conforme Sorrigueta-Hernández et al. (2020) as esportistas que foram objeto de estudo não apresentavam doença ou quadro patológico concomitante. Em atletas femininas de elite, em geral, a fisioterapia contribuiu para o tratamento de continência urinária mais do que em mulheres controle. Em atletas de elite do sexo feminino, ex-atletas de elite do sexo feminino e em mulheres grávidas que se envolvem regularmente em atividades aeróbicas, a fisioterapia apresenta melhores resultados no controle da IU. Sobre esse fator, verificase que a prevalência da IU ocorre de acordo com as modalidades esportivas que as mulheres praticam, de forma que o CrossFit se destaca como sendo a modalidade mais mencionada pelos autores.

Kari et al. (2021) constatou-se que não houve diferenças estatisticamente significativas na nova IU de início entre os grupos ou quando o colapso da nova IU de início nos grupos de intervenção em comparação com os controles. Após a intervenção por meio de fortalecimento do assoalho pélvico durante doze semanas, o estudo concluiu que não ocorreu mudança estatisticamente significativa na IU após o treinamento de força. Por essa razão, os autores sugerem que pacientes com IU sejam submetidas a protocolos de tratamento prolongados, com mais de 12 semanas.

Conforme Bógea et al. (2020) verificou-se que a intervenção intensificada por meio de treinamento dos músculos do assoalho pélvico em mulheres com IUE pode apresentar ganhos significativos na qualidade de vida dessas mulheres. As mulheres foram positivas sobre ambas as intervenções, a adesão a ambas as intervenções foi semelhante e ambos as intervenções foram facilitadas pelo desejo de melhorar a incontinência urinária e dificultadas pela falta de tempo.

Para Hagen et al. (2020) o treinamento dos músculos do assoalho pélvico pelo método biofeedback foi não significativamente mais caro do que o treinamento muscular básico do assoalho pélvico, mas também não estava associado a significativamente melhora da qualidade de vida de pacientes mulheres com IUE. Conforme o estudo de Mazur-Bialy et al. (2020) em estágios avançados da IU a fisioterapia é uma das formas de tratamento mais importantes no preparo dos pacientes para intervenção cirúrgica, podendo aumentar de forma significativa o sucesso da cirurgia.

A fisioterapia melhora significativamente a qualidade de vida de mulheres com IU. Para os autores, cabe ressaltar a relevância dos protocolos de tratamento a partir de biofeedback e eletroestimulação, agnetostimulação e treinamentos de vibração, de forma que esses tratamentos podem ser utilizados para conter sintomas de IU em mulheres. Aponta-se também que, em determinadas situações, recomenda-se a combinação de várias técnicas fisioterapêuticas, de forma que biofeedback e eletroestimulação, agnetostimulação e treinamentos de vibração são as terapias mais comumente usadas para o tratamento da IU (Mazur-Bialy et al., 2020).

Por outro lado, conforme Rzymski et al. (2021) os mecanismos mais adequados para o tratamento contrações caudais e para o controle adequado da musculatura abdominal devem evitar pressão intraabdominal excessiva. De acordo com o estudo, a ultrassonografia do assoalho pélvico é o único método objetivo para o exame do assoalho pélvico entre esportistas e uma ferramenta que deve ser usada rotineiramente por urofisioterapeutas e uroginecologistas no tratamento de IU.

Frigo et al. (2015) sinaliza a importância de serem realizadas intervenções em mulheres diagnosticadas com IUE por meio da fisioterapia, podendo direcionar o tratamento de mulheres com IUE para que essas possam obter mais qualidade de vida e bem estar. Nessa perspectiva, esse estudo corrobora com as pesquisas que apontam para a relação entre atividades físicas de alto impacto com a incidência de IUE. Por sua vez, não existe consenso na literatura sobre fatores que podem aumentar as chances de a incontinência ocorrer (Bógea et al., 2020).

Como demonstrado pelos autores Frigo et al. (2015), Hagen et al. (2020), Mazur-Bialy et al. (2020), Rzymski et al. (2021) o tratamento da IUE se relaciona diretamente ao fortalecimento de músculos do assoalho pélvico. Nesse entendimento, a intervenção por meio da fisioterapia é relatada como uma das formas de promover qualidade de vida e bem estar para mulheres com IUE. Ressalta-se que a literatura não contraindica a prática 
de atividades físicas de alto impacto. Todavia, reitera-se a necessidade de mulheres que praticam esportes em alto nível devem buscar trabalhar a musculatura perineal como método preventivo.

Diante do exposto, salienta-se que popularidade crescente de exercícios de alto impacto (Zago; Silves; Lopes, 2018) decorre na necessidade de que exista divulgação de conhecimentos entre praticantes de atividades físicas, assim como profissionais da saúde que atuam diante de cuidados e assistência relacionada a IUE. Nesse sentido, as mulheres que vivenciam esta patologia devem ser encorajadas a buscar por terapia adequada, minimizando as complicações relacionadas a incontinência urinária e suas implicações indesejáveis na vida da mulher. Cabe ressaltar a importância do autoconhecimento de mulheres sobre a saúde da mulher e sobre a importância do fortalecimento do assoalho pélvico, assim como sobre fatores como percepção de esforço e consciência corporal, aspectos essenciais para a sustentação do assoalho pélvico diante da prática de atividades físicas de alto impacto (Silva et al., 2007).

O acolhimento deve ser tido como uma prioridade para os serviços de assistência de saúde, de forma que esses serviços devem considerar as características da população acometida por IUE assim como a legislação brasileira. Para tal, devem estar preparados para abordar questões do gênero feminino, rompendo com a precariedade no atendimento à saúde da mulher (Brasil, 2004).

$\mathrm{O}$ direito à saúde ainda é muito distante do ideal no Brasil, existindo burocracias diversas que tornam dificultoso o processo de acesso ao tratamento adequado. Por sua vez, o Sistema de Saúde deve ter como objetivo assistir à mulher em várias etapas da sua vida. Nesse viés, as políticas de saúde até o presente momento são insuficientes no que tange ao atendimento sobre a IU em mulheres (Medeiros et al., 2005).

\section{Considerações finais}

A partir do estudo realizado, não é possível afirmar que como causa direta da IUE tem-se a prática de exercícios de alto impacto. Entretanto, é possível apontar correlação entre mulheres praticantes de atividades físicas com impacto e a incidência de deste tipo de incontinência, em casos nos quais estas mulheres não pratiquem atividades que têm o objetivo de fortalecer os músculos do assoalho pélvico, fator que estaria intimamente associado à IU. Dentre outros aspectos que podem contribuir para a predisposição de incontinência urinária, conforme a literatura, é possível apontar que esportes como a modalidade CrossFit, BodyPump e Ginástica Rítmica vêm sendo estudados com maior recorrência. Por sua vez, os estudos não relacionam diretamente o risco da prática desses esportes com a prevalência de da incontinência urinária de esforço.

Não existem tratamentos convencionados e totalmente indicados para essa patologia em mulheres do sexo feminino que pratiquem esportes. Por sua vez, o treinamento de fortalecimento de músculos do assoalho pélvico foi relatado nos estudos com taxas promissoras para o controle de IUE. O treinamento dos músculos do assoalho pélvico não tem efeitos adversos conhecidos e deve ser oferecido às mulheres.

Nesse sentido, é importante que exista aumento da conscientização sobre as disfunções do assoalho pélvico e o potencial para o tratamento eficaz da IU em mulheres que praticam determinados esportes e/ou atividades físicas de impacto. A fisioterapia é certamente um elemento importante no tratamento da incontinência urinária de esforço. Em estágios avançados da doença, entretanto, a fisioterapia é um elemento importante no preparo do paciente para o tratamento cirúrgico, aumentando significativamente o sucesso da cirurgia. Além disso, a fisioterapia melhora significativamente a qualidade de vida das mulheres.

\section{Referência}

Abrams, P., Cardozo, L., Fall, M., Griffiths, D., Rosier, P., Ulmsten, U., ... \& Wein, A. 2003. The standardisation of terminology in lower urinary tract function: report from the standardisation sub-committee of the International Continence Society. Urology, 61(1), 37-49.

Almeida, P. P. D., \& Machado, L. R. G. 2012. A prevalência de incontinência urinária em mulheres praticantes de jump. Fisioterapia em Movimento, 25, 55-65.

Araujo, M. P. D., Parmigiano, T. R., Negra, L. G. D., Torelli, L., Carvalho, C. G. D., Wo, L., ... \& Sartori, M. G. F. 2015. Avaliação do assoalho pélvico de atletas: existe relação com a incontinência urinária?. Revista Brasileira de Medicina do Esporte, 21, 442-446.

Azevedo, R. M. F. 2013. Eficácia do treino dos músculos do pavimento pélvico no tratamento da incontinência urinária de esforço em jovens atletas: um estudo clínico randomizado controlado. Dissertação de Mestrado em Fisioterapia Materno-Infantil apresentada na Faculdade de Ciências da Saúde. Porto, PT: Universidade Fernando Pessoa. 
Borba, A. M. C. D., Lelis, M. A. D. S., \& Brêtas, A. C. P. 2008. Significado de ter incontinência urinária e ser incontinente na visão das mulheres. Texto \& Contexto-Enfermagem, 17, 527-535.

Bogéa, M., Gomes, P., Dinnucci, A., Dutra, F., \& Zaidan, P. 201). Incontinência urinária de esforço em mulheres praticantes de Crossfit: Um estudo transversal de prevalência. International Journal of Development Research, 8(7), 21642-5.

Campbell, K. G., Batt, M. E., \& Drummond, A. 2020. A feasibility study of the physiotherapy management of urinary incontinence in athletic women: trial protocol for the POsITIve study. Pilot and Feasibility Studies, 6(1), 1-11.

Brasil. 2004. Política de qualificação da atenção à saúde. Brasília, DF: Ministério da Saúde.

Caetano, A. S., Tavares, M. D. C. G. C. F., \& Lopes, M. H. B. D. M. 2007. Incontinência urinária e a prática de atividades físicas. Revista brasileira de medicina do esporte, 13, 270-274.

Casey, E. K., \& Temme, K. 2017. Pelvic floor muscle function and urinary incontinence in the female athlete. The Physician and sportsmedicine, 45(4), 399-407.

Frigo, L. F., Bordin, D. F., \& Romeiro, C. A. P. 2015. Avaliação da frequência de incontinência urinária em jogadoras de voleibol amadoras de Santa Maria-RS. Cinergis, 16(4).

Hannestad, Y. S., Rortveit, G., Sandvik, H., \& Hunskaar, S. 2000. A community-based epidemiological survey of female urinary incontinence: The Norwegian EPINCONT Study. Journal of clinical epidemiology, 53(11), 1150-1157.

Haylen, B. T., De Ridder, D., Freeman, R. M., Swift, S. E., Berghmans, B., Lee, J., ... \& Schaer, G. N. 2010. An International Urogynecological Association (IUGA)/International Continence Society (ICS) joint report on the terminology for female pelvic floor dysfunction. Neurourology and Urodynamics: Official Journal of the International Continence Society, 29(1), 4-20.

Higar, R., Lopes, M. H. B., \& Reis, M. J. 2008. Risk factors for urinary incontinence in womem. Revista da Escola de Enfermagem da USP, 42(1).

Henkes, D. F., Fiori, A., Carvalho, J. A. M., Tavares, K. O., \& Frare, J. C. 2015. Incontinência urinária: o impacto na vida de mulheres acometidas e o significado do tratamento fisioterapêutico. Semina: ciências Biológicas e da Saúde, 36(2), 45-56.

Sorrigueta-Hernández, A., Padilla-Fernandez, B. Y., Marquez-Sanchez, M. T., Flores-Fraile, M. C., FloresFraile, J., Moreno-Pascual, C., ... \& Lorenzo-Gomez, M. F. 2020. Benefits of physiotherapy on urinary incontinence in high-performance female athletes. Meta-analysis. Journal of Clinical Medicine, 9(10), 3240.

Hagen, S., Bugge, C., Dean, S. G., Elders, A., Hay-Smith, J., Kilonzo, M., ... \& Williams, L. R. 2020. Basic versus biofeedback-mediated intensive pelvic floor muscle training for women with urinary incontinence: the OPAL RCT. Health Technology Assessment, 24(70), 1.

Kari, B., \& Gram. M. C. D. 2020. High level rhythmic gymnasts and urinary incontinence: Prevalence, risk factors, and influence on performance. Scand J Med Sci Sports, 30(1).

Khaida, L. S. 2018. Prevalência e fatores de risco da incontinência urinária em atletas de diferentes modalidades desportivas: Revisão bibliográfica. Projeto e Estágio Profissionalizante II. Licenciatura em Fisioterapia. Porto, PT: Universidade Fernando Pessoa.

Kari, B., Haakstad, L A. H., Paulsen, G., \& Rustaden, A. M. 2021. Does regular strength training cause urinary incontinence in overweight inactive women? A randomized controlled trial. International Urogynecology Journal. 32(1), 1-8.

Khowailed, I. A., Pinjuv-Turney, J., Lu, C., \& Lee, H. 2020. Stress Incontinence during Different High-Impact Exercises in Women: A Pilot Survey. International Journal of Environmental Research and Public Health, $17(22), 8372$.

Kargar, M., Mohammadalipour, Z., Doosti, A., Lorzadeh, S., \& Japoni-Nejad, A. 2014. High prevalence of class 1 to 3 integrons among multidrug-resistant diarrheagenic Escherichia coli in southwest of Iran. Osong public health and research perspectives, 5(4), 193-198.

Leal, L. O., Alves Santos, M., de Freitas Santos, N. M., Magalhães Braga, L., \& de Faria Nunes, K. C. 2020. Prevalência de incontinência urinária e perfil miccional de mulheres praticantes de Crossfit ${ }^{\circledR}$. Fisioterapia Brasil, 21(2).

Martins, L. A., Santos, K. M. D., Dorcínio, M. B. A., Alves, J. O., Roza, T. D., \& Luz, S. C. T. D. 2017. A perda de urina é influenciada pela modalidade esportiva ou pela carga de treino? Uma revisão sistemática. Revista Brasileira de Medicina do Esporte, 23, 73-77. 
Mazur-Bialy, A. I., Kołomańska-Bogucka, D., Nowakowski, C., \& Tim, S. 2020. Urinary incontinence in women: modern methods of physiotherapy as a support for surgical treatment or independent therapy. Journal of clinical medicine, 9(4), 1211.

Medeiros, V. C. R. D., Medeiros, R. C. D., Moraes, L. M. D., Menezes Filho, J. B. D., Ramos, E. S. N., \& Saturnino, A. C. R. D. 2005. Câncer de colo de útero: análise epidemiológica e citopatológica no estado do Rio Grande do Norte. Revista Brasileira de Análises Clínicas, 37(4), 227-31.

Nygaard, I. E., \& Shaw, J. M. 2016. Physical activity and the pelvic floor. American journal of obstetrics and gynecology, 214(2), 164-171.

Nygaard, I., Girts, T., Fultz, N. H., Kinchen, K., Pohl, G., \& Sternfeld, B. 2005. Is urinary incontinence a barrier to exercise in women?. Obstetrics \& Gynecology, 106(2), 307-314.

Polit, D. F., \& Beck, C. T. 2006. Using research in evidence-based nursing practice. Essentials of nursing research. Methods, appraisal and utilization. Philadelphia, US: Lippincott Williams \& Wilkins.

Patrizzi, L. J., Viana, D. A., Silva, L. M. A., \& Pegori, M. S. 2014. Incontinência urinária em mulheres jovens praticantes de exercício físico. Revista Brasileira de Ciência e Movimento, 105-110.

Padilha, J., da Silva, A. C., Mazo, G. Z., \& de Godoy Marques, C. M. 2018. Investigação da qualidade de vida de mulheres com incontinência urinária. Arquivos de Ciências da Saúde da UNIPAR, 22(1).

Rzymski, P., Burzyński, B., Knapik, M., Kociszewski, J., \& Wilczak, M. (2021). How to balance the treatment of stress urinary incontinence among female athletes?. Archives of Medical Science: AMS, 17(2), 314.

Rådmark, O., Werz, O., Steinhilber, D., \& Samuelsson, B. 2015. 5-Lipoxygenase, a key enzyme for leukotriene biosynthesis in health and disease. Biochimica et Biophysica Acta (BBA)-Molecular and Cell Biology of Lipids, 1851(4), 331-339.

Saboia, D. M., Firmiano, M. L. V., Bezerra, K. D. C., Vasconcelos, J. A., Oriá, M. O. B., \& Vasconcelos, C. T. M. 2017. Impacto dos tipos de incontinência urinária na qualidade de vida de mulheres. Revista da Escola de Enfermagem da USP, 51.

Santos, A. M., Silveira, H. R. M., Lima, T. V. S., \& Pontes, I. E. A. 2020. Ocorrência da incontinência urinária em praticantes de esportes de alto impacto: uma revisão integrativa da literatura. Revista Temas em Saúde, 20(2).

Silva, A. G., de Carvalho, R. R. C., de Almeida Ferreira, S., Valença, M. P., da Silva Filho, J. C., \& Santos, I. C. R. V. (2020). Incontinência urinária em mulheres: fatores de risco segundo tipo e gravidade. Cogitare enfermagem, 25.

Silva, E., Sarreta, F. O., \& Bertani, I. F. 2007. As políticas públicas de saúde no Brasil: o Sistema Único de Saúde (SUS) e a rede de saúde em Franca. Serviço Social \& Realidade, 81-103.

Tibana, R. A., Almeida, L. M., \& Prestes, J. 2015. Crossfit ${ }^{\circledR}$ riscos ou benefícios? O que sabemos até o momento? Revista Brasileira Ciência em Movimento, 23(1), 182-5.

Wikander, L., Kirshbaum, M., \& Gahreman, D. E. 2020. Urinary Incontinence and Women CrossFit Competitors. International Journal of Women's Health,1(12).

Zago, G. L., Silva, C. A., \& Lopes, C. A. M. 2018. Incontinência Urinária de Esforço na prática de atividade física em mulheres e os exercícios de KEGEL. Anais do Fórum de Iniciação Científica do UNIFUNEC, 8(8).

\section{Minicurrículo}

Luciana Gomes de Souza Camelo. Fisioterapeuta, Faculdade Paranaense, Curitiba, Paraná, Brasil.

Raphael Fernando Soares Gonçalves. Fisioterapeuta, Faculdade Paranaense, Curitiba, Paraná, Brasil.

Tânia Aparecida Barbosa Rzniski. Fisioterapeuta, Faculdade Paranaense, Curitiba, Paraná, Brasil. 
Como citar: Camelo, L.G.S., Gonçalves, R.F.S., Rzniski, T. 2021. Exercícios físicos de alto impacto e incontinência urinária em mulheres jovens: revisão integrativa da literatura. Pubsaúde, 7, a241. DOI: https://dx.doi.org/10.31533/pubsaude7.a241

Recebido: 11 ago. 2021.

Revisado e aceito: 24 ago. 2021.

Conflito de interesse: os autores declaram, em relação aos produtos e companhias descritos nesse artigo, não ter interesses associativos, comerciais, de propriedade ou financeiros que representem conflito de interesse.

Licenciamento: Este artigo é publicado na modalidade Acesso Aberto sob a licença Creative Commons Atribuição 4.0 (CC-BY 4.0). 